\title{
A Strange Case of Left Bowel Ischemia after Right Hernioplasty
}

\author{
Girolamo Geraci Franco Pisello Giuseppe Modica \\ Francesco Li Volsi Massimo Cajozzo Carmelo Sciumè \\ Section of General and Thoracic Surgery, University of Palermo, Palermo, Italy
}

\section{Key Words}

Inguinal hernia $\cdot$ Complications $\cdot$ Colonic ischemia

\begin{abstract}
We report the first observed case of a young man who suffered of large and unsuspected left bowel ischemia following an elective right open hernioplasty. A 54-year-old man had a 2-year history of right inguinal reducible mass and was admitted to hospital for an elective day case open inguinal hernioplasty for a direct right inguinal hernia. Apart from mild hypertension controlled with ACE inhibitor, he was medically fit and well. The patient was submitted to open tension-free mesh repair with polypropylene preshaped mesh with local infiltration anesthesia and additive sedation with midazolam. The local anesthesia and surgery were uneventful and he was discharged home on the same day as per day case protocol. He was readmitted about $12 \mathrm{~h}$ after discharge with a history of central and left lower abdominal pain with palpable mass, and distension and fever $\left(38^{\circ} \mathrm{C}\right)$. After imaging and laboratory studies the patient was submitted to explorative surgery with the suspicion of left colonic ischemia. After intraoperative confirmation we performed standard left hemicolectomy. The postoperative course was uneventful; the patient was discharged in good general condition on the 7th postoperative day. Actually, the patient is in follow-up, with normal coagulation and hemochromocytometric pattern, asymptomatic for hypercholesterolemia and atrial flutter/fibrillation. Complications relating to bowel during open techniques of hernia repair are limited to two situations: the freeing of an incarcerated or strangulated segment of bowel and inadvertent laceration of large bowel in the presence of a sliding hernia. Following this strange case of colonic ischemia, a boolean Medline search (terms: hernia, complication, repair, groin, herniorrhaphy, hernioplasty, all major MESH subjects without language restriction) revealed no previous similar cases reported. However, to our knowledge, there is another trouble hypothesis: not causality but casualty. In conclusion, to our knowledge this is the first reported case of large left bowel ischemia following right open hernioplasty. We can conclude that the presence of a dolichocolon is an added risk factor for this rare and uneventful complication, but further investigations and case reports are necessary to estabilish the real causality.
\end{abstract}




\begin{tabular}{|c|c|c|c|}
\hline Gastroenterolay & DOI: $10.1159 / 000260072$ & & $\begin{array}{l}\text { ISSN 1662-0631 } \\
\text { www.karger.com/crg }\end{array}$ \\
\hline
\end{tabular}

\section{Introduction}

We report the case of a young man who suffered of large left bowel ischemia following an elective right open hernioplasty. After a careful review of the literature, we can conclude that this is the first such case to be described.

\section{Case Report}

A 54-year-old man had a 2-year history of right $3 \mathrm{~cm}$ inguinal reducible mass and was admitted to hospital for an elective day case open inguinal hernioplasty for a symptomatic direct right inguinal hernia, type IIIA according to the classification of Nyhus [1]. He was an ex-smoker who used to smoke 15 cigarettes a day but had quit smoking 24 months earlier. His ASA grade was II. Apart from mild hypertension controlled with ACE inhibitor, he was medically fit and well.

The patient was submitted to open tension-free mesh repair with polypropylene preshaped mesh (Surgipro $^{\mathrm{TM}}$ Mesh, Auto Suture ${ }^{\mathrm{TM}}$, UK, Type 1 according to the classification of Amid) [2] with local infiltration anesthesia and additive sedation with midazolam $(0.05-0.1 \mathrm{mg} / \mathrm{kg}$ i.v., in $20 \mathrm{ml}$ of isotonic $\mathrm{NaCl}$ 0.9\% solution). The local anesthesia and surgery were uneventful and he was discharged home on the same day as per day case protocol. He was readmitted about $12 \mathrm{~h}$ after discharge with a history of central and left lower abdominal pain with palpable mass, and distension and fever $\left(38^{\circ} \mathrm{C}\right)$. Digital exploration of the rectal ampulla revealed normal stools. After $2 \mathrm{~h}$, though his vital signs were normal, the abdominal pain persisted with cutaneous hyperesthesia in the left lower abdomen. His hematology and biochemistry results revealed leukocytosis (20,470 WBC, NV 4,000-10,000) and anemia $(2,100,000$ RBC, NV 4,000,000-6,000,000). His chest radiograph was normal, and a supine abdominal film showed a gas-filled transverse colon without any unusual features (however, a negative plain film should not exclude ischemia of the colon). Abdominal CT revealed dolichosigmoid with thickened wall, mesosigmoid hyperdensity with, in its context, $400 \mathrm{ml}$ of mixed blood and clot collection and perisplenic effusion (fig. 1). There was neither free air in peritoneal cavity nor air-fluid levels.

As preoperative care, the patients was stabilized using i.v. fluids, antibiotics covering the colonic flora, nasogastric tube decompression, and bladder catheterization. Blood was available. With the strong suspicion of sigmoid ischemic infarction, the patient was submitted to explorative laparotomy. We confirmed the presence of massive mesosigmoid agglomerate of clot with signs of ischemic suffering of the serosal layer and, after rinsing of the peritoneal cavity, we performed left hemicolectomy and mechanical end-to-end colorectal anastomosis. We did not observe signs of bleeding or perforation in the peritoneal layer of right hernioplasty (fig. 2 , fig. 3 ).

Postoperative hematology and biochemistry results were normal and the postoperative course was uneventful; the patient was discharged in good general condition on the 7th postoperative day. At present the patient is in cardiovascular and surgical follow-up, with normal coagulation and hemochromocytometric pattern, asymptomatic for hypercholesterolemia and atrial flutter/fibrillation.

\section{Discussion}

Much has been written on the complications of herniorrhaphy and hernioplasty and the modern-day hernia surgeon, whether a general surgeon or a hernia zealot, will have developed his or her own approach to preoperative consent, the type of procedure and anesthesia undertaken, and subsequent follow-up [3,4]. Actually, the complications of open groin hernioplasty are well defined (table 1).

Local anesthesia allows surgery in unfit patients, rapid mobilization and ambulatory surgery and does not require expensive preoperative laboratory work-up, but requires a gentler touch, is limited by toxic doses of local agents and may require a second operator to administer sedation before and after analgesia [4]. Complications relating to bowel during open techniques of hernia repair are limited to two situations: (1) the freeing of an incarcerated or strangulated segment of bowel and (2) inadvertent laceration of large bowel in the presence of a sliding hernia. The site of strangulation is usually the 
superficial inguinal ring. In these cases, mortality has ranged from 6-23\% [3]. The publication by Ryan [5] has conclusively supported the new attitude that opening of a sac is not necessary, that the high ligation of a sac is unnecessary, and that the countless, complicated maneuvers to reperitonealize bowel and abdominal cavity are confusing, possibly dangerous, and should be discarded in favor of simple reduction of the hernia sac and contents into the preperitoneal space.

Insufficient blood perfusion to the colon may result from arterial occlusion by embolus or thrombosis (AMAE or AMAT), thrombosis of the venous system (MVT), or nonocclusive processes. Embolic phenomena account for approximately $50 \%$ of all cases, arterial thrombosis for about $25 \%$, NOMI for roughly $20 \%$, and MVT for less than $10 \%$. Hemorrhagic infarction is the common pathologic pathway whether the occlusion is arterial or venous [6]. Injury severity is inversely proportional to the mesenteric blood flow and is influenced by the number of vessels involved, systemic mean pressure, duration of ischemia, and collateral circulation. The superior mesenteric vessels are involved more frequently than the inferior mesenteric vessels, with blockage of the latter often being silent because of better collateral circulation [7].

The occlusive syndrome of the inferior mesenteric artery (IMA) is uncommon and generally due to thrombosis from atherosclerotic disease. Formation of collateral vessels, from the colic branches of the SMA and from the hemorrhoidal arteries, branches of the hypogastric arteries, are often not effective to prevent an acute colorectal ischemia due to occlusion of the IMA at the origin [8]. Damage to the affected bowel portion may range from reversible ischemia to transmural infarction with necrosis and perforation. The injury is complicated by reactive vasospasm in the SMA region after the initial occlusion. Arterial insufficiency causes tissue hypoxia, leading to initial bowel wall spasm. This leads to gut emptying by vomiting or diarrhea. Mucosal sloughing may cause bleeding into the gastrointestinal tract. At this stage, little abdominal tenderness is usually present, producing the classic intense visceral pain disproportionate to physical examination findings [9].

In our opinion, on the basis of technique of hernioplasty and CT, there are two etiopathogenetical hypotheses: first, a volvulus with fulcrum on mesosigmoid, caused by manipulation of the right peritoneal cavity; second, a systemic effect of first puncture of local anesthesia (plexus blocking too deep). Also low-flow states cause peripheral vasodilation and shunting of the blood from the gut to the periphery; cases of patients without atherosclerotic disease of their mesenteric arteries having ischemia have been reported, but this is rare and occurs in patients with profound hypovolemic shock. Furthermore, digitalis has been found to cause vasoconstriction of both arterial and venous smooth muscle cells in the mesenteric vasculature: of patients with acute mesenteric ischemia, $20-30 \%$ have nonocclusive disease [10]. Other causes of nonocclusive mesenteric ischemia are use of vasopressive drugs, ergotamine, cocaine and digitalis, but our patient had neither use of these drugs nor referred postoperative hypovolemia [11].

Early diagnosis is important to improve survival rates, since diagnosis may be overlooked because of the vague nature of the patients' symptoms; in most cases of late or missed diagnosis, mortality from intestinal infarction is very high, from 60 to $90 \%$ [8]. Actually, in our opinion, the mechanism by which this patient experienced intestinal ischemia is poorly understood, but we are considering the opportunity to modify our informed consenst schedule after this event. 
Following this strange case of colonic ischemia, a boolean Medline search (terms: hernia, complication, repair, groin, herniorrhaphy, hernioplasty, all major MESH subjects without language restriction) revealed no previous similar cases reported. However, to our knowledge, there is another trouble hypothesis: not causality but casualty. Many other reports are necessary in the future to establish the real cause of this phenomenon.

\section{Conclusions}

To our knowledge this is the first reported case of large left bowel ischemia following right open hernioplasty. We can conclude that the presence of a dolichocolon is an added risk factor for this rare and uneventful complication, but further investigations and case reports are necessary to establish the real causality. This event will give us the opportunity to modify our informed consenst schedule.

Table 1. Morbidity of inguinal hernia repair $[3,4]$

\begin{tabular}{|c|c|c|c|c|c|}
\hline & Operative & & Early & & Later ( $>5$ years) \\
\hline Incision & $\begin{array}{l}\text { Bruising, } \\
\text { hematoma }\end{array}$ & $10 \%$ & Infection & $0-9 \%$ & $\begin{array}{l}\text { Chronic sepsis and }<1 \% \\
\text { sinus formation }\end{array}$ \\
\hline Scrotum & & & Genital edema, orchitis/atrophy & $<1 \%$ & \\
\hline Open technique & $\begin{array}{l}\text { Nerve and vascular } \\
\text { injuries }\end{array}$ & $2-20 \%$ & Hydrocele & $<1 \%$ & $\begin{array}{l}\text { Recurrence } \\
\text { Groin pain }\end{array}$ \\
\hline Systemic & $\begin{array}{l}\text { General/local } \\
\text { anesthesia }\end{array}$ & $6 \%$ & $\begin{array}{l}\text { Chest infection, urinary } \\
\text { retention and venous thrombosis }\end{array}$ & $<1 \%$ & \\
\hline
\end{tabular}




\begin{tabular}{r|l|l|l} 
Case Repports in & $\begin{array}{l}\text { Case Rep Gastroenterol 2010;4:12-18 } \\
\text { Dol: 10.1159/000260072 }\end{array}$ & Published online: February 3, 2010 & $\begin{array}{l}\text { O 2010 S. Karger AG, Basel } \\
\text { ISSN 1662-0631 } \\
\text { www.karger.com/crg }\end{array}$ \\
\hline
\end{tabular}

Fig. 1. CT finding.
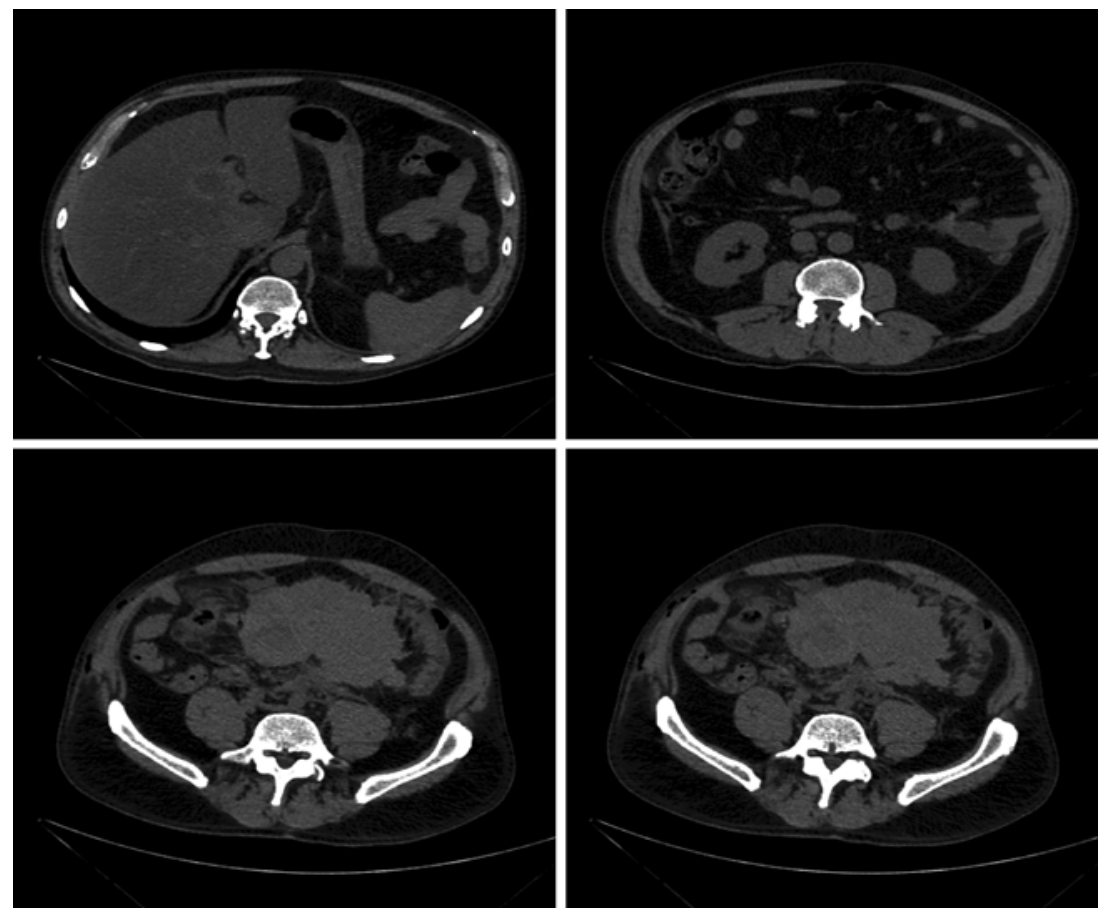

Fig. 2. Intraoperative mesosigma infarction.
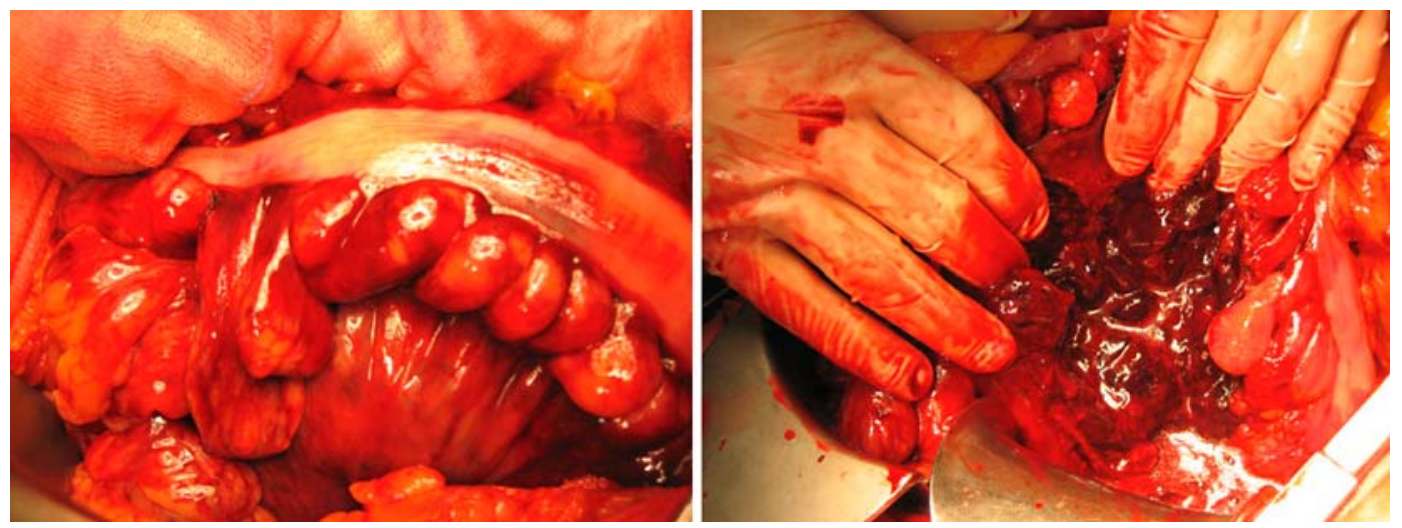
Case Reports in | Case Rep Gastroenterol 2010;4:12-18 Gastruenterology DOI: $10.1159 / 000260072$

Fig. 3. Surgical extraperitoneal control of right herniotomy.

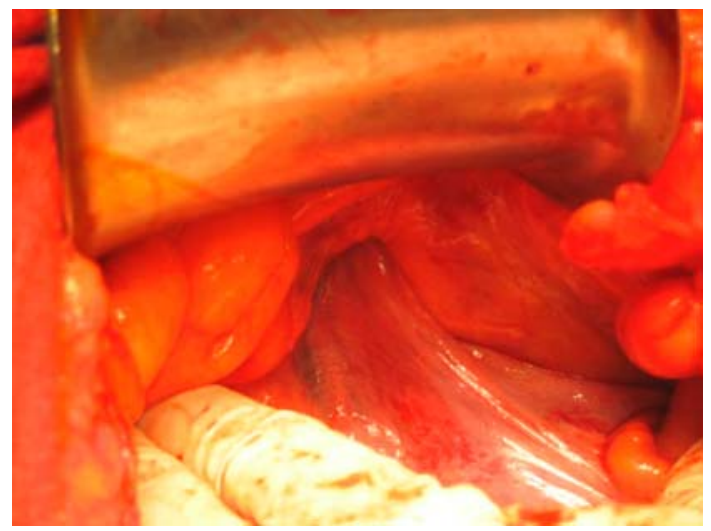




\section{References}

1 Nyhus LM: Individualization of hernia repair: a new era. Surgery 1993;114:1-2.

2 Amid PK: Groin hernia repair: open techniques. World J Surg 2005;29:10461051.

-3 Bendavid R: Complications of groin hernia surgery. Surg Clin North Am 1998;78:1089-1103.

4 Stephenson BM: Complications of open groin hernia surgery. Surg Clin North Am 2003;83:1255-1278.

5 Ryan EA: An analysis of 313 consecutive cases of indirect sliding inguinal hernias. Surg Gynecol Obstet 1956;102:45-58.

6 Kozuch PL, Brandt LJ: Review article: diagnosis and management of mesenteric ischaemia with an emphasis on pharmacotherapy. Aliment Pharmacol Ther 2005;21:201-215.

7 Rosenblum JD, Boyle CM, Schwartz LB: The mesenteric circulation. Anatomy and physiology. Surg Clin North Am 1997;77:289-306.

8 Romano S, Lassandro F, Scaglione M, Romano L, Rotondo A, Grassi R: Ischemia and infarction of the small bowel and colon: spectrum of imaging findings. Abdom Imaging 2006;31:277-292.

-9 Fink S, Chaudhuri TK, Davis HH: Acute mesenteric ischemia and malpractice claims. South Med J 2000;93:210-214.

10 Yasuhara H: Acute mesenteric ischemia: the challenge of gastroenterology. Surg Today 2005;35:185-195.

11 Brandt LJ, Boley SJ: AGA technical review on intestinal ischemia. American Gastrointestinal Association. Gastroenterology 2000;118:954-968. 